\title{
Boosting for Learning Multiple Classes with Imbalanced Class Distribution
}

\author{
Yanmin Sun \\ Department of Electrical \\ and Computer Engineering \\ University of Waterloo \\ Waterloo, Ontario, Canada \\ y8sun@engmail.uwaterloo.ca
}

\author{
Mohamed S. Kamel \\ Department of Electrical \\ and Computer Engineering \\ University of Waterloo \\ Waterloo, Ontario, Canada \\ mkamel@pami.uwaterloo.ca
}

\author{
Yang Wang \\ Pattern Discovery \\ Software Systems Ltd. \\ 554 Parkside Drive \\ Waterloo, Ontario, Canada \\ yang@patterndiscovery.com
}

\begin{abstract}
Classification of data with imbalanced class distribution has posed a significant drawback of the performance attainable by most standard classifier learning algorithms, which assume a relatively balanced class distribution and equal misclassification costs. This learning difficulty attracts a lot of research interests. Most efforts concentrate on bi-class problems. However, bi-class is not the only scenario where the class imbalance problem prevails. Reported solutions for bi-class applications are not applicable to multi-class problems. In this paper, we develop a cost-sensitive boosting algorithm to improve the classification performance of imbalanced data involving multiple classes. One barrier of applying the cost-sensitive boosting algorithm to the imbalanced data is that the cost matrix is often unavailable for a problem domain. To solve this problem, we apply Genetic Algorithm to search the optimum cost setup of each class. Empirical tests show that the proposed cost-sensitive boosting algorithm improves the classification performances of imbalanced data sets significantly.
\end{abstract}

\section{Introduction}

Classification is an important task of knowledge discovery in databases (KDD) and data mining. Recently, reports from both academy and industry indicate that the imbalanced class distribution of a data set has posed a serious difficulty to most classifier learning algorithms which assume a relatively balanced distribution [9,12]. Imbalanced class distribution is characterized as that there are many more instances of some classes than others. With imbalanced data, classification rules that predict the small classes tend to be fewer and weaker than those that predict the prevalent classes; consequently, test samples belonging to the small classes are misclassified more often than those belonging to the prevalent classes. Standard classifiers usually perform poorly on imbalanced data sets because they are designed to generalize from training data and output the simplest hypothesis that best fits the data. Therefore, the simplest hypothesis pays less attention to rare cases. However, in many cases, identifying rare objects is of crucial importance; classification performances on the small classes are the main concerns in determining the property of a classification model.

The difficulty raised by the class imbalance problem with both academic research and practical applications in the community of machine learning and data mining attracts a lot of research interests. Reported works focus on three aspects of the class imbalance problem: 1) what are the proper evaluation measures of classification performance in the presence of the class imbalance problem? 2) what is the nature of the class imbalance problem, i.e. in what domains do class imbalances most hinder the performance of a standard classifier? [9]; and 3) what are the possible solutions in dealing with the class imbalance problem? With regard to the first aspect, it is stated that accuracy is traditionally the most commonly used measure in both assessing the classification models and guiding the search algorithms. However, for a classification model induced from a data set with imbalanced class distribution, accuracy is no longer a proper measure since rare classes have very few impact on accuracy than prevalent classes [11]. Some other evaluation measures, such as recall, precision, F-measure, G-mean and Receiver Operation Characteristic (ROC) Curve Analysis, are then explored and proposed as more proper evaluation measures $[1,10,16]$. With respect to the second aspect, a thorough study can be found in [9]. Other relevant works are reported in $[10,23,24]$. These studies show that the imbalanced class distribution is not the only factor that hinders the classification performance. Other factors that deteriorate the performance include the training sample size, the separability and the presences of sub-concepts within a group. The third aspect is the focus of most publications ad- 
dressing the class imbalance problem. Almost all reported solutions are designed for the bi-class scenario.

In a bi-class application, the imbalanced problem is observed as one class is represented by a large amount of samples while the other is represented by only a few. The class with very few training samples and usually associated with high identification importance, is referred as the positive class; the other one as the negative class. The learning objective of this kind of data is to obtain a satisfactory identification performance on the positive (small) class. Reported solutions for the bi-class applications can be categorized as data level and algorithm level approaches [2]. At the data level, the objective is to re-balance the class distribution by re-sampling the data space including oversampling instances of the positive class and undersampling instances of the negative class, sometimes, uses the combination of the two techniques [2]. At the algorithm level, solutions try to adapt the existing classifier learning algorithms to bias towards the positive class, such as cost sensitive learning [15] and recognition based learning [8]. In addition to these solutions, another approach is boosting. Boosting algorithms change the underlying data distribution and apply the standard classifier learning algorithms to the revised data space iteratively. From this point of view, boosting approaches should be categorized as solutions at data level.

The AdaBoost (Adaptive Boosting) algorithm [5, 19] is reported as an effective boosting algorithm to improve classification accuracies of any "weak" learning algorithms. It weighs each sample reflecting its importance and places the most weights on those examples which are most often misclassified by the preceding classifiers. This forces the following learning to concentrate on those samples hard to be correctly classified. When the AdaBoost algorithm is adapted to tackle the class imbalance problem, advantages are: 1) it is applicable to most classifier learning algorithms; 2) the sample weighting strategy of the AdaBoost algorithm is equivalent to re-sampling the data space combining both up-sampling and down-sampling; 3) as a resampling method, AdaBoost updates the data space automatically eliminating the extra learning cost for exploring the optimal class distribution; and 4) resampling through weighting samples has little information loss and the AdaBoost algorithm is stated to be immune to overfitting [6]. Boosting is therefore an attractive technique in tackling the class imbalance problem. Within the bi-class applications, some variants of the AdaBoost algorithm in tackling the imbalance problem are reported, such as AdaCost [4], CSB1 and CSB2 [21], RareBoost [11] and AdaC1, AdaC2 and AdaC3 [20]. These boosting algorithms inherit the general learning framework of the AdaBoost algorithm and feed misclassification costs into the weight update formula of AdaBoost to distinguish the uneven learning importance between classes. As these algorithms use cost items, they are also regarded as cost-sensitive boosting algorithms.

Yet bi-class is not the only scenario where the class imbalance problem prevails. In practice, most applications have more than two classes where the unbalanced class distributions hinder the classification performance. Solutions for bi-class problems are not applicable directly to multiclass cases. One possible solution is to convert a multi-class problem into a number of bi-class problems, i.e., classifying each individual class versus all the other classes. The obvious drawbacks of this treatment are: 1) to learn an identification model for each class is expensive in training; 2) results of each class label assignment are not comparable due to the decision can be made differently for different classes; and 3) one class versus the other classes will worse the imbalanced distribution even more for the small classes. Even though cost-sensitive boosting algorithms can be adopted for multiple class applications, research efforts are still limited to bi-class cases. One reason is that the cost matrix is often unavailable for a given problem domain. For cost-sensitive learning and/or measures, the costmatrix is assumed known for different types of errors or samples. Without the cost matrix, experiments for bi-class problems were conducted by set up a range of cost factors manually. Such a strategy is not applicable to multiple class cases since to figure out satisfactory cost values manually for multiple classes is a non-trivial job. Hence, searching a efficient cost setup becomes a critical issue for applying the cost-sensitive boosting approach to multiple class applications. To our knowledge, there is no reported work on the class imbalance problem addressing multiple class cases.

In this paper, we develop a cost-sensitive boosting algorithm for the class imbalance problem in the scenario of multiple classes. We extended the original AdaBoost algorithm to multi-class cases. The straightforward generalization is called AdaBoost.M1 [5]. By using the same inference methods provided in $[5,18]$, we prove that the upper bound error of the final hypothesis output by AdaBoost.M1 holds the same format as that by AdaBoost. Thus, the crucial weight update parameter of AdaBoost.M1 is selected as the same as that of AdaBoost. Among those reported cost-sensitive boosting algorithms, we select and extend AdaC2 [20] to multi-class cases. The extension inheriting the framework of AdaBoost.M1 is therefore denoted as AdaC2.M1. We then compare the weighting strategy of AdaC2.M1 with that of AdaBoost.M1 to explore the boosting efficiency of AdaC2.M1. To decide the cost setups that can be applied to the AdaC2.M1 algorithm, we apply Genetic Algorithm (GA) which achieves outstanding performance in finding optimal parameters. To evaluate the performance, three "real world" data sets are tested since their classification performances are hindered by their imbalanced class distributions. 
Given: $\left(x_{1}, y_{1}\right), \cdots,\left(x_{m}, y_{m}\right)$ where $x_{i} \in X, y_{i} \in Y=$ $\{-1,+1\}$

Initialize $D^{1}(i)=1 / m$.

For $t=1, \cdots, T$ :

1. Train base learner $h_{t} \rightarrow Y$ using distribution $D^{t}$

2. Choose weight updating parameter: $\alpha_{t}$

3. Update and normalize sample weights:

$$
D^{t+1}(i)=\frac{D^{t}(i) \exp \left(-\alpha_{t} h_{t}\left(x_{i}\right) y_{i}\right)}{Z_{t}}
$$

Where, $Z_{t}$ is a normalization factor.

Output the final classifier:

$$
H(x)=\operatorname{sign}\left(\sum_{t=1}^{T} \alpha_{t} h_{t}(x)\right)
$$

\section{Figure 1. AdaBoost Algorithm}

\section{AdaBoost Algorithm}

The original AdaBoost algorithm reported in [5, 19] takes as input a training set $\left\{\left(x_{1}, y_{1}\right), \cdots,\left(x_{m}, y_{m}\right)\right\}$ where each $x_{i}$ is an n-tuple of attribute values belonging to a certain domain or instance space $X$, and $y_{i}$ is a label in a label set $Y=\{-1,+1\}$ in the context of bi-class applications. The Pseudocode for AdaBoost is given in Figure 1.

It has been shown in [19] that the training error of the final classifier is bounded as

$$
\frac{1}{m}\left|\left\{i: H\left(x_{i}\right) \neq y_{i}\right\}\right| \leq \prod_{t} Z_{t}
$$

Let

$$
f(x)=\sum_{t=1}^{T} \alpha_{t} h_{t}(x)
$$

By unraveling the update rule of Equation 1, we have that

$$
D^{t+1}(i)=\frac{\exp \left(-\sum_{t} \alpha_{t} h_{t}\left(x_{i}\right) y_{i}\right)}{m \prod_{t} Z_{t}}=\frac{\exp \left(-y_{i} f\left(x_{i}\right)\right)}{m \prod_{t} Z_{t}}
$$

By the definition of the final hypothesis of Equation 2 , if $H\left(x_{i}\right) \neq y_{i}$, the $y_{i} f\left(x_{i}\right) \leq 0$ implying that $\exp \left(-y_{i} f\left(x_{i}\right)\right) \geq 1$. Thus,

$$
\left[H\left(x_{i}\right) \neq y_{i}\right] \leq \exp \left(-y_{i} f\left(x_{i}\right)\right) .
$$

where for any predicate $\pi$,

$$
[\pi]= \begin{cases}1 & \text { if } \pi \text { holds } \\ 0 & \text { otherwise }\end{cases}
$$

Combining Equation 4 and 5 gives the error upper bound of Equation 3 since

$$
\begin{aligned}
\frac{1}{m} \sum_{i}\left[H\left(x_{i}\right) \neq y_{i}\right] & \leq \frac{1}{m} \sum_{i} \exp \left(-y_{i} f\left(x_{i}\right)\right) \\
= & \sum_{i}\left(\prod_{t} Z_{t}\right) D^{t+1}(i)=\prod_{t} Z_{t}
\end{aligned}
$$

To minimize the error upper-bound, on each boosting round, the learning objective is to minimize

$$
\begin{aligned}
Z_{t} & =\sum_{i} D^{t}(i) \exp \left(-\alpha_{t} y_{i} h_{t}\left(x_{i}\right)\right) \\
& =\sum_{i} D^{t}(i)\left(\frac{1+y_{i} h_{t}\left(x_{i}\right)}{2} e^{-\alpha}+\frac{1-y_{i} h_{t}\left(x_{i}\right)}{2} e^{\alpha}\right)
\end{aligned}
$$

Then, by minimizing $Z_{t}$ on each round, $\alpha_{t}$ is induced as

$$
\alpha_{t}=\frac{1}{2} \log \left(\frac{\sum_{i, y_{i}=h_{t}\left(x_{i}\right)} D^{t}(i)}{\sum_{i, y_{i} \neq h_{t}\left(x_{i}\right)} D^{t}(i)}\right)
$$

The sample weight updating goal of AdaBoost is to decrease the weight of training samples which are correctly classified and increase the weights of the opposite part. Therefore, $\alpha_{t}$ should be a positive value demanding that the training error should be less than randomly guessing (0.5) based on the current data distribution. That is

$$
\sum_{i, y_{i}=h_{t}\left(x_{i}\right)} D^{t}(i)>\sum_{i, y_{i} \neq h_{t}\left(x_{i}\right)} D^{t}(i)
$$

\section{AdaBoost.M1 Algorithm}

The original AdaBoost algorithm is designed for bi-class applications. There are several methods of extending AdaBoost to the multi-class cases. The straightforward generalization one, called AdaBoost.M1 in [5], is adequate when the base learner is effective enough to achieve reasonably high accuracy (training error should be less than 0.5).

AdaBoost.M1 differs slightly from AdaBoost. The main differences are the replacements of the weight update formula of Equation 1 by:

$$
D^{t+1}(i)=\frac{D^{t}(i) \exp \left(-\alpha_{t} I\left[h_{t}\left(x_{i}\right)=y_{i}\right]\right)}{Z_{t}}
$$

where, $Z_{t}$ is a normalization factor, and

$$
I\left[h_{t}\left(x_{i}\right)=y_{i}\right]= \begin{cases}+1 & \text { if } h_{t}\left(x_{i}\right)=y_{i} \\ -1 & \text { if } h_{t}\left(x_{i}\right) \neq y_{i}\end{cases}
$$

and the final hypothesis of Equation 2 by: 


$$
H(x)=\arg \max _{C_{i}}\left(\sum_{t=1}^{T} \alpha_{t}\left[h_{t}(x)=C_{i}\right]\right)
$$

By using the same inference methods provided in $[5,18]$, we can prove the following bound still holds on the training error of the final hypothesis output $H(x)$ (Equation 15) by AdaBoost.M1:

$$
\frac{1}{m}\left|\left\{i: H\left(x_{i}\right) \neq y_{i}\right\}\right| \leq \prod_{t} Z_{t}
$$

where

$$
Z_{t}=\sum_{i} D^{t}(i) \exp \left(-\alpha_{t} I\left[h_{t}\left(x_{i}\right)=y_{i}\right]\right)
$$

To prove this theorem, we reduce the setup for AdaBoost.M1 to an instantiation of AdaBoost. For clarity, variables in the reduced AdaBoost space are marked with tildes. For each of the given samples $\left(x_{i}, y_{i}\right)$, an AdaBoost sample $\left(\tilde{x}_{i}, \tilde{y}_{i}\right)$ is generated, where $\tilde{x}_{i}=x_{i}$ and $\tilde{y}_{i}=1$, i.e., each AdaBoost sample has label 1. The AdaBoost distribution $\tilde{D}$ over samples is set to be equal to the AdaBoost.M1 distribution $D$. On each round, an AdaBoost hypothesis $\tilde{h}_{t}$ is defined as

$$
\tilde{h}_{t}\left(x_{i}\right)=I\left[h_{t}\left(x_{i}\right)=y_{i}\right]= \begin{cases}+1 & \text { if } h_{t}\left(x_{i}\right)=y_{i} \\ -1 & \text { if } h_{t}\left(x_{i}\right) \neq y_{i}\end{cases}
$$

and

$$
\tilde{f}\left(x_{i}\right)=\sum_{t=1}^{T} \alpha_{t} \tilde{h}_{t}(x)
$$

Suppose the AdaBoost.M1's final hypothesis $H(x)$ makes a mistake on instance $\left(x_{i}, y_{i}\right)$ so that $H\left(x_{i}\right) \neq y_{i}$. Then, by the definition the final hypothesis of Equation 15,

$$
\sum_{t=1}^{T} \alpha_{t}\left[h\left(x_{i}\right)=y_{i}\right] \leq \sum_{t=1}^{T} \alpha_{t}\left[h_{t}\left(x_{i}\right)=H\left(x_{i}\right)\right]
$$

This implies

$$
\sum_{t=1}^{T} \alpha_{t}\left[h\left(x_{i}\right)=y_{i}\right] \leq \frac{1}{2} \sum_{t=1}^{T} \alpha_{t}
$$

and

$$
\sum_{t=1}^{T} \alpha_{t}\left[h\left(x_{i}\right) \neq y_{i}\right] \geq \frac{1}{2} \sum_{t=1}^{T} \alpha_{t}
$$

Then,

$$
\tilde{f}\left(x_{i}\right)=\sum_{t=1}^{T} \alpha_{t} I\left[h\left(x_{i}\right)=y_{i}\right]
$$

$$
\begin{aligned}
& =\sum_{t=1}^{T} \alpha_{t}\left[h\left(x_{i}\right)=y_{i}\right]-\sum_{t=1}^{T} \alpha_{t}\left[h\left(x_{i}\right) \neq y_{i}\right] \\
& \leq 0
\end{aligned}
$$

implying that $\exp \left(-\tilde{f}\left(x_{i}\right)\right) \geq 1$. Thus

$$
\left[H\left(x_{i}\right) \neq y_{i}\right] \leq \exp \left(-\tilde{f}\left(x_{i}\right)\right) .
$$

Thus, by using the same inference method of AdaBoost, we can get the stated bound on training error (Equation 16). To minimize the the error upper-bound, $Z_{t}$ is minimized on each round. $\alpha_{t}$ is induced the same as Equation 11. To make $\alpha_{t}$ a positive value, each weak hypothesis has training error less than $1 / 2$ as stated by the Equation 12 .

\section{AdaC2.M1 Algorithm}

Suppose we have $k$ classes and $m$ samples. Let $c(i, j)$ denote the cost of misclassifying an example of class $i$ to the class $j$. In all cases, $c(i, j)=0.0$ for $i=j$. Let $c(i)$ denote the cost of misclassifying samples of class $i$. $c(i)$ is usually derived from $c(i, j)$. There are many possible rules for the derivation, among which one form suggested in [22] is:

$$
c(i)=\sum_{j}^{k} c(i, j) .
$$

Moreover, we can easily expand this class-based cost to sample-based cost. We take the misclassification cost stand for the recognition importance respecting to each class. Hence for samples in the same class, their misclassification costs can be set with the same value. Suppose that the $i^{\text {th }}$ sample belongs to class $j$. We associate this sample with a misclassification cost $c_{i}$ which equals to the misclassification cost of class $j$, i.e., $c_{i}=c(j)$.

AdaC2.M1 inherits the general learning framework of the AdaBoost.M1 algorithm except that it feeds costs into the weight update formula (Equation 13) of AdaBoost.M1 as:

$$
D^{t+1}(i)=\frac{c_{i} D^{t}(i) \exp \left(-\alpha_{t} I\left[h_{t}\left(x_{i}\right)=y_{i}\right]\right)}{Z_{t}}
$$

Unravelling the weight update rule of Equation 26, we obtain

$$
\begin{aligned}
D^{t+1}(i) & =\frac{c_{i}^{t} \exp \left(-\sum_{t} \alpha_{t} I\left[h_{t}\left(x_{i}\right)=y_{i}\right]\right)}{m \prod_{t} Z_{t}} \\
& =\frac{c_{i}^{t} \exp \left(-I\left[h_{t}\left(x_{i}\right)=y_{i}\right]\right)}{m \prod_{t} Z_{t}}
\end{aligned}
$$

where $c_{i}^{t}$ stands for $c_{i}$ to the power of $t$, and 


$$
Z_{t}=\sum_{i} c_{i} D^{t}(i) \exp \left(-\alpha_{t} I\left[h_{t}\left(x_{i}\right)=y_{i}\right]\right)
$$

By using the same inference methods of AdaBoost.M1, we can prove the training error of the final classifier is bounded as:

$$
\frac{1}{m}\left|\left\{i: H\left(x_{i}\right) \neq y_{i}\right\}\right| \leq \prod_{t} Z_{t} \sum_{i} \frac{c_{i} D^{t}(i)}{c_{i}^{t+1}} \leq \frac{1}{\gamma} \prod_{t} Z_{t}
$$

Where $\gamma$ is a constant that $\forall i, \gamma<c_{i}^{t+1}$. Thus the learning objective on each round is to find $\alpha_{t}$ to minimize $Z_{t}$ (Equation 29). $\alpha_{t}$ is then selected taking costs into consideration as:

$$
\alpha_{t}=\frac{1}{2} \log \frac{\sum_{i, y_{i}=h_{t}\left(x_{i}\right)} c_{i} D^{t}(i)}{\sum_{i, y_{i} \neq h_{t}\left(x_{i}\right)} c_{i} D^{t}(i)}
$$

To ensure that the selected value of $\alpha_{t}$ is positive, the following condition should hold:

$$
\sum_{i, y_{i}=h_{t}\left(x_{i}\right)} c_{i} D^{t}(i)>\sum_{i, y_{i} \neq h_{t}\left(x_{i}\right)} c_{i} D^{t}(i)
$$

\section{Resampling Effects}

In a multi-class application of $k$ classes, the confusion matrix through a classification process can be presented in Table 1 . Where $C_{i}$ denotes the class label of the $i^{\text {th }}$ class.

Table 1. Confusion Matrix

\begin{tabular}{cc|cccc} 
& & Predicted & class & & \\
& & $C_{1}$ & $C_{2}$ & $\ldots . \cdot$ & $C_{k}$ \\
\hline True & $C_{1}$ & $n_{11}$ & $n_{12}$ & $\ldots . \cdot$ & $n_{1 k}$ \\
class & $C_{2}$ & $n_{21}$ & $n_{22}$ & $\ldots . \cdot$ & $n_{2 k}$ \\
& $\cdot$ & $\cdot$ & $\cdot$ & $\cdot$ & $\cdot$ \\
& $\cdot$ & $\cdot$ & $\cdot$ & $\cdot$ & $\cdot$ \\
& $\cdot$ & $\cdot$ & $\cdot$ & $\cdot$ & $\cdot$ \\
& $C_{k}$ & $n_{k 1}$ & $n_{k 2}$ & $\ldots . \cdot$ & $n_{k k}$ \\
\hline
\end{tabular}

The general weighting strategy of AdaBoost.M1 is to increase weights of false predictions and decrease those of true predictions. Let $T P$ denote the true predictions and $F P$ the false predictions of a classification output. Referring to the confusion matrix of Table 1 , for class $i$, the true prediction number, denoted by $T P(i)$, equals to $n_{i i}$ and the false prediction number, $F P(i)$, equals to $\sum_{j=1, j \neq i}^{k} n_{i j}$. Thus, $T P=\sum_{i=1}^{k} T P(i)=\sum_{i=1}^{k} n_{i i}$ and $F P=$ $\sum_{i=1}^{k} F P(i)=\sum_{i=1}^{k} \sum_{j=1, j \neq i}^{k} n_{i j}$. It has been shown that after weights being updated by AdaBoost.M1, sample distributions on these two parts get to even. AdaC2.M2 adapts AdaBoost.M1's weighting strategy by inducing the cost items. In this section, we will explore the weight updating mechanisms of both AdaBoost.M1 and AdaC2.M1. Our interest focuses on the resampling effect of AdaC2.M1.

\section{$5.1 \quad$ AdaBoost.M1}

Based on the inference in [19], $\alpha$ is selected to minimize $Z$ as a function of $\alpha$ (Equation 17). The first derivative of $Z$ is

$$
\begin{aligned}
Z_{t}^{\prime}(\alpha) & =\frac{d Z}{d \alpha} \\
& =-\sum_{i} D^{t}(i) I\left[h_{t}\left(x_{i}\right)=y_{i}\right] \exp \left(-\alpha_{t} I\left[h_{t}\left(x_{i}\right)=y_{i}\right]\right) \\
& =-Z_{t} \sum_{i} D^{t+1}(i) I\left[h_{t}\left(x_{i}\right)=y_{i}\right]
\end{aligned}
$$

by definition of $D^{(t+1)}$ (Equation 13). To minimize $Z_{t}, \alpha_{t}$ is selected such that $Z^{\prime}(\alpha)=0$, i.e.,

$$
\begin{aligned}
& \sum_{i} D^{t+1}(i) I\left[h_{t}\left(x_{i}\right)=y_{i}\right] \\
= & \sum_{i, h_{t}\left(x_{i}\right)=y_{i}} D^{t+1}(i)-\sum_{i, h_{t}\left(x_{i}\right) \neq y_{i}} D^{t+1}(i)=0
\end{aligned}
$$

That is:

$$
\sum_{i, h_{t}\left(x_{i}\right)=y_{i}} D^{t+1}(i)=\sum_{i, h_{t}\left(x_{i}\right) \neq y_{i}} D^{t+1}(i)
$$

Hence, after weights being updated, weight distributions on misclassified samples and correctly classified samples get to even, i.e., $T P=F P$. This will make the learning of next iteration the most difficult [6].

By the weight update formula of AdaBoost.M1 (Equation 13), weights of samples in two groups specified to class $i, \mathrm{TP}(\mathrm{i})$ and $\mathrm{FP}(\mathrm{i})$, updated from the $t^{\text {th }}$ iteration to the $(t+1)^{t h}$ iteration can be summarized as $T P_{t+1}(i)=$ $T P_{t}(i) / e^{\alpha_{t}}$ and $F P_{t+1}(i)=F P_{t}(i) \cdot e^{\alpha_{t}}$. With $\alpha_{t}$ being a number of positive, identical for all classes, weights of false predictions (FP) are improved equally; weights of true predictions (TP) are decreased equally, i.e., weighting scheme of AdaBoost.M1 treats samples of different classes equally.

To illustrate this weighting effect, we take an example. Suppose we have a data set of three classes. The sample distribution after a classification process is presented in Figure 2(a). The left side represents correctly classified samples which occupy a larger proportion of the space 


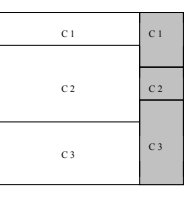

(a)

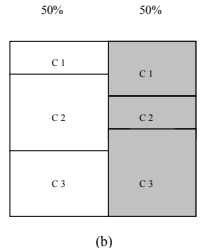

Figure 2. Resampling Effects of AdaBoost.M1

and the right side shaded represents samples mis-classified. On each side, samples are grouped by class labels, i.e., $\mathrm{C} 1, \mathrm{C} 2$ and $\mathrm{C} 3$. By weighting and normalizing of AdaBoost.M1, correctly classified space shrinks and misclassified space expands until these two parts get to equal. Figure 2 (b) demonstrates this result. The notable point is on each part, correctly classified and misclassified, each group (class) shrinks or expands at the same ratio. Observationally, the classes with relatively more misclassified samples will get expanded, which are not necessary the classes we care about. To strengthen the learning on the "weak" classes, we expect more weighted sample sizes on them.

\section{$5.2 \quad$ AdaC2.M1}

The learning objective of AdaC2.M1 algorithm is to select $\alpha_{t}$ for minimizing $Z_{t}$ on each round (Equation 30). The first derivative of $Z_{t}$ as a function of $\alpha_{t}$ is

$$
\begin{aligned}
Z_{t}^{\prime}(\alpha) & =\frac{d Z}{d \alpha} \\
& =-\sum_{i} c_{i} D^{t}(i) I\left[h_{t}\left(x_{i}\right)=y_{i}\right] \exp \left(-\alpha_{t} I\left[h_{t}\left(x_{i}\right)=y_{i}\right]\right) \\
& =-Z_{t} \sum_{i} D^{t+1}(i) I\left[h_{t}\left(x_{i}\right)=y_{i}\right]
\end{aligned}
$$

by definition of $D^{(t+1)}$ (Equation 26). To minimize $Z_{t}, \alpha_{t}$ is selected such that $Z^{\prime}(\alpha)=0$. The unique solution for $\alpha_{t}$ is presented by Equation 31. By the definition of $D^{t+1}$ (Equation 26), for the next iteration we will have

$$
\sum_{i, h_{t}\left(x_{i}\right)=y_{i}} D^{t+1}(i)=\sum_{i, h_{t}\left(x_{i}\right) \neq y_{i}} D^{t+1}(i)
$$

It indicates that weight of the correctly classified group and that of the misclassified group get to even after sample weights being updated by AdaC2.M1, i.e., $T P=F P$. Same as AdaBoost.M1, this weighting result will make the learning of next iteration the most difficult.

By the weight update formula of AdaC2.M1( Equation 26), sample weights of two groups respecting to class $i$, $T P(i)$ and $F P(i)$, updated from the $t^{t h}$ iteration to the $(t+1)^{t h}$ iteration can be summarized as $T P_{t+1}(i)=c(i)$.
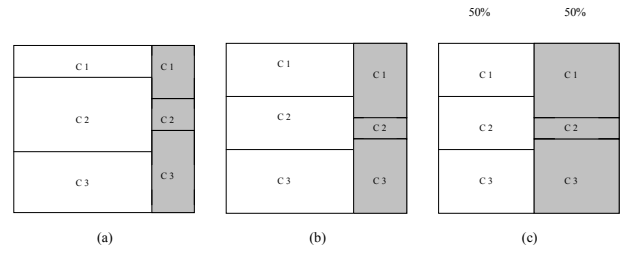

Figure 3. Resampling Effects of AdaC2.M1

$T P_{t}(i) / e^{\alpha_{t}}$ and $F P_{t+1}(i)=c(i) \cdot F P_{t}(i) \cdot e^{\alpha_{t}}$. Where $c(i)$ denotes the misclassification cost of class $i$. This weighting process can be interpreted in two steps. At the first step, each sample, no matter in which groups (TP or FP), is first weighted by its cost item (which equals to the misclassification cost of the class that the sample belongs to). Samples of the classes with larger cost values will obtain more sample weights, on the other side, samples of the classes with smaller cost values will lose their sizes. Consequently, the class with the largest cost value will always enlarge its class size at this phase. The second step is actually the weighting procedure of AdaBoost.M1, i.e., weights of false predictions are expanded and those of true predictions are shrunk. The expanding or shrinking ratio for samples of all classes is the same.

To demonstrate this weighting process, we use the same example as illustrated for AdaBoost.M1. In this case, we associate each class with a misclassification cost. Suppose the costs are 3, 1 and 2 respecting to class $C 1, C 2$ and $C 3$. Each sample obtains a cost value according to its class label. Let the sample distribution after a classification process presented in Figure 3(a) be the same with that presented in Figure 2(a). By the weighting strategy of AdaC2.M1, the first step is to reweight each sample by its cost item. After normalizing, classes with relative larger cost values are expanded, oppositely, the other class is shrunk. In our example, class sizes of class $C 1$ and $C 3$ are increased and class size of class $C 2$ is decreased as presented in Figure 3 (b). At the next step, correctly classified space shrinks and misclassified space expands until these two parts get to even. If we compare Figure 3(c) with Figure 2 (b), obviously, we can find out that class $C 1$ expands its class size updated by AdaC2.M1 more than that updated by AdaBoost.M1.

This observation shows that we can use the cost values to adjust the data distributions among classes. For those classes with poor performances, we can associate them with relative higher cost values such that relatively more weights are accumulated on those parts. As a result, learning will bias and more relevant samples might be identified. However, if weights are over boosted, more irrelevant samples can be included simultaneously. Precision values of these classes and recall values of the other classes will be decreased. Hence, how to figure out an efficient cost 
setup which is able to yield satisfactory classification performance is the next problem to be solved.

\section{Searching for an Optimum Cost Setup by Genetic Algorithm}

Genetic Algorithm (GA) is a directed random search technique invented by Holland [7]. It is based on the theory of natural selection and evolution. GA is a robust search method requiring little information to search in a large search space. Generally, GA requires two elements for a given application: 1) Encoding of candidate solutions; and 2) Fitness function for evaluating the relative performance of candidate solutions to identify the better one.

Genetic Algorithm codes candidate solutions of the search space as binary strings of fixed length. It employs a population of strings initialized at random, which evolve to the next generation by genetic operators such as selection, crossover and mutation. The fitness function evaluates the quality of solutions. GA tends to take advantage of the fittest solutions by giving them greater weight, and concentrating the search in the regions which lead to better solutions of the problem. Therefore, GA searches through a population of points in contrast to the single point of focus of most other search algorithms, such as Simulated Annealing and Hill Climbing algorithms. Though it might not find the best solution, it would come up with a partially optimal solution.

In our case, we employ GA for searching an optimal misclassification cost setup, which will be applied to the AdaC2.M1 algorithm trying to improve the classification performance of the unbalanced data sets. Let $c(i)$ denote the misclassification cost of class $i$. Then cost items of $k$ classes making up a cost vector of $k$ elements $[c(1) c(2) \cdots c(k)]$ can be encoded as a binary string. The fitness value of each vector is the measurement of the classification performance when the vector is integrated in the AdaC2.M1 algorithm applied to a base classification system. Evaluation of the classification performance depends on the learning objective. According to the learning objectives, the fitness function is varied. The final output of GA is a vector that yields the most satisfactory classification performance among all tests.

\section{Experiments}

In this section, we set up experiments to investigate the cost-sensitive boosting algorithm AdaC2.M1 respecting to its capability in dealing with the class imbalance problem with multiple classes. For this purpose, we apply both AdaBoost.M1 and AdaC2.M1 to decision tree classification system C4.5 [17]. Then their performances on data sets with multiple classes where the unbalanced class distributions hinder the classification performances are compared and analyzed. Three data sets, Car data, New-thyroid data, and Nursery data, are taken from UCI Machine Learning Database [14] for our experiments.

\subsection{Leaning Objectives and Evaluation Measures}

Refer to the confusion matrix of Table 1, the true prediction of the $i^{t h}$ class is the number of $n_{i i}$. Classification accuracy is then calculated as:

$$
\text { Accuracy }=\frac{\sum_{i=1}^{k} n_{i i}}{\sum_{i, j=1}^{k} n_{i j}}
$$

The evaluation measure of accuracy is inadequate in reflecting the classifier's performance on classifying each single class, especially on those small classes. The learning objective with unbalanced data with multiple classes can be either to improve the recognition success on a specific class or to balance identify ability over every classes. Respecting to the different learning objectives, the classification performance is evaluated by different measures. Regarding to classifying a single class, one should consider both its ability of recognizing available samples and the accuracy of recognizing relevant samples. These two aspects are referred to as recall and precision in information retrieval. Let $R_{i}$ and $P_{i}$ denote recall and precision of class $C_{i}$ respectively, then $R_{i}$ and $P_{i}$ are defined as:

$$
R_{i}=\frac{n_{i i}}{\sum_{j=1}^{k} n_{i j}}
$$

and

$$
P_{i}=\frac{n_{i i}}{\sum_{j=1}^{k} n_{j i}}
$$

Clearly neither of these measures are adequate by themselves. F-measure $(\mathrm{F})$ is suggested in [13] to integrate these two measures as an average:

$$
F_{i}-\text { measure }=\frac{2 R_{i} P_{i}}{R_{i}+P_{i}}
$$

It is obvious that if the F-measure is high when both the recall and precision should be high.

When the performances of all classes are interested, classification performance of each class should be equally represented in the evaluation measure. For the bi-class scenario, Kubat et al [12] suggested the G-mean as the geometric means of recall values of two classes. Expanding this measure to the multiple class scenario, we define G-mean as the geometric means of recall values of every classes: 


$$
G-\text { mean }=\left(\prod_{i=1}^{k} R_{i}\right)^{1 / k}
$$

As each recall value representing the classification performance of a specific class is equally accounted, G-mean is capable to measure the balanced performance among classes of a classification output.

Another often used method for evaluating classification of unbalanced data is ROC analysis [1]. A ROC graph depicts relative trade-offs between true positives and false positives within the bi-class concepts. The ROC analysis method needs a classifier to yield a score representing the degree to which an example pertaining to a class. In this study, we will evaluate our experimental performances by F-measure and G-mean.

\subsection{Experiment Method}

For each data set, available samples are used for both cost setup searching and performance evaluation. For this purpose, we carry on two sections of partitions. The first section of partitions is for searching cost setups by GA. The data set is randomly divided into two sets: $80 \%$ as the training set and the remaining $20 \%$ as the validation set for measuring the goodness of a bunch of cost setups generated by GA. The out put is a cost vector which obtains the best fitness value among all tests. This process is repeated 20 times such that one prototype is obtained from a pool of 20 cost vectors. The second section of partitions, totally independent from the first section, is for evaluating the classification performance. The whole data set is repartitioned into two sets: $80 \%$ as the training set and the remaining $20 \%$ as the test set. This process is repeated 10 times to obtain an average performance. For a consistent comparison, classification models of C4.5, C4.5 applied by AdaBoost.M1 and C4.5 applied by AdaC2.M2 are trained and evaluated with same data partitions.

The cost setup used by AdaC2.M2 is the prototype from the validation tests with the first section of partitions. In our experiments, we take the mean of a pool of 20 cost vectors as the cost setup prototype. As stated in [3], given a set of cost setups, the decisions are unchanged if each one in the set is multiplied by a positive constant or added with a constant. The ratios among cost values denote the deviations of the leaning importance among classes. Therefore, normalizing each cost vector in the pool is a necessary step before calculating the mean value. The normalization method is, first, to set the value of the element with the maximum value in a vector as 1 , then, to scale other elements' values in the vector with the ratio of 1 over the maximum value. After normalizing of each cost vector, a mean is calculated as the prototype. The prototype vector is also normalized before putting it in use for further experiments.

\subsection{Car Evaluation Database}

This database was derived for car evaluating. There are 1728 instances with each is described by 6 nominal ordered attributes. The whole data are grouped into 4 classes. Table 2 describes the class distribution.

Table 2. Class Distribution

\begin{tabular}{cccc}
\hline \hline index & class name & class size & class distribution \\
\hline C1 & unacc & 1210 & $70.023 \%$ \\
C2 & acc & 384 & $22.222 \%$ \\
C3 & good & 69 & $3.993 \%$ \\
C4 & v-good & 65 & $3.762 \%$ \\
\hline \hline
\end{tabular}

Class C3 and C4 are two small classes which posses only $3.993 \%$ and $3.762 \%$ samples respectively. We first use the second section of data partitions to test the performance of C4.5 and C4.5 applied by AdaBoost.M1. For this part of experiments, the classification performance are recorded respecting to both each individual class and the overall performance. Respecting to each class F-measure value (Equation 38) is calculated. Respecting to the overall performance, both classification accuracy (Equation 35) and G-mean (Equation 39) are reported. Experiment results are tabulated in Table 3.

Table 3. Performance of C4.5 \& AdaBoost.M1

\begin{tabular}{cccc}
\hline \hline Measure & Class & C4.5 & AdaBoost.M1 \\
\hline F & C1 & 0.9754 & 0.9753 \\
measure & C2 & 0.8817 & 0.8873 \\
& C3 & 0.7097 & 0.7781 \\
& $\mathrm{C} 4$ & 0.7486 & 0.8845 \\
\hline Accuracy & & 0.9344 & 0.9440 \\
G-mean & & 0.8336 & 0.8758 \\
\hline \hline
\end{tabular}

Respecting to the classification of $\mathrm{C} 4.5$, performances of classes $\mathrm{C} 1$ and $\mathrm{C} 2$ are significantly better than those of class $\mathrm{C} 3$ and $\mathrm{C} 4$, which are two small classes. By applying AdaBoost.M1, performances of class $\mathrm{C} 1$ and $\mathrm{C} 2$ remain similar values; that of class $\mathrm{C} 4$ is significantly improved; performance of class C3 is also improved but far behind other classes'. As for the overall performance, classification accuracy of C4.5 is improved slightly; G-mean value is improved by $4.22 \%$ by applying AdaBoost.M1. Based on these observations, for testing AdaC2.M1 we set up two learning objectives: 1) to further balance the identify ability on each class; and 2) to improve the recognition ability of class C3. We then run GA for searching an efficient cost setup with data partitions of the first section. 
Respecting to the first learning objective, the fitness function is G-mean evaluation. The resulting prototype of a cost vector is [0.3281 0.66820 .78491 .0000$]$. Integrating this cost vector into AdaC2.M1, classification performances are evaluated by G-mean on the data partitions of the second section such that C4.5, C4.5 applied by AdaBoost.M1, and C4.5 applied by AdaC2.M1 are evaluated on the same training and test partitions. Table 4 tabulates results for comparisons.

Table 4. G-mean Evaluation

\begin{tabular}{cccc}
\hline \hline Class & C4.5 & AdaBoost.M1 & AdaC2.M1 \\
\hline C1(R) & 0.9637 & 0.9707 & 0.9586 \\
C2(R) & 0.9083 & 0.9056 & 0.9459 \\
C3(R) & 0.7175 & 0.7395 & 0.8540 \\
C4(R) & 0.7902 & 0.9151 & 0.9139 \\
G-mean & 0.8336 & 0.8758 & 0.9146 \\
\hline \hline
\end{tabular}

As G-mean is calculated as the geometric means of recall values of every classes, each row indicated by class label in Table 4 are the recall values achieved by C4.5, C4.5 applied by AdaBoost.M1, and C4.5 applied by AdaC2.M1 respectively and the row indicated by "G-mean" are those G-mean values. Respecting to the two small classes C3 and $\mathrm{C} 4$, recall value of $\mathrm{C} 4$ is improved and that of $\mathrm{C} 3$ fails to be improved by AdaBoost.M1. By applying AdaC2.M1, recall value of $\mathrm{C} 3$ is greatly improved. In general, G-mean value of C4.5 is increased by $4.22 \%$ by applying AdaBoost.M1 and by $8.10 \%$ by applying AdaC2.M1, which obtains the highest $\mathrm{G}$-mean values through increasing recall values of both classes $\mathrm{C} 3$ and $\mathrm{C} 4$.

Respecting to the second learning objective, the fitness function is the F-measure evaluation of class $\mathrm{C} 3$. The resulting prototype cost vector is [0.5412 0.82170 .75361 .0000$]$. Integrating this cost vector to AdaC2.M1, classification performance of class $\mathrm{C} 3$ is evaluated by the data partitions of the second section. Table 5 presents the performances of C4.5, AdaBoost and AdaC2.M1 including recall (R), precision $(\mathrm{P})$ and $\mathrm{F}$-measure $(\mathrm{F})$ values of class $\mathrm{C} 3$. F-measure value of $\mathrm{C} 4.5$ is improved by AdaBoost.M1 through increasing the precision value. By applying AdaC2.M1, both recall and precision values are improved and keep relatively even. AdaC2.M1 hence achieves the best F-measure value.

Table 5. F-measure Evaluation on Class C3

\begin{tabular}{cccc}
\hline \hline & C4.5 & AdaBoost.M1 & AdaC2.M1 \\
\hline $\mathrm{R}$ & 0.7175 & 0.7395 & 0.8364 \\
$\mathrm{P}$ & 0.7068 & 0.8389 & 0.8289 \\
$\mathrm{~F}$ & 0.7097 & 0.7781 & 0.8304 \\
\hline \hline
\end{tabular}

\subsection{New-Thyroid Database}

The goal of this data set is to predict a patient's thyroid to the class euthyroidism (normal), hypothyroidism or hyperthyroidism. This data is a simple database containing 215 instances of patients, each described by 5 attributes. Table 6 describes the class distribution.

Table 6. Class Distribution

\begin{tabular}{cccc}
\hline \hline index & class name & class size & class distribution \\
\hline C1 & normal & 150 & $69.77 \%$ \\
C2 & hyper & 35 & $16.28 \%$ \\
C3 & hypo & 30 & $13.95 \%$ \\
\hline \hline
\end{tabular}

Two classes of this data set, $\mathrm{C} 2$ and $\mathrm{C} 3$, are small classes. By the same experiment method as described previously for the Car data, we first test C4.5 and C4.5 applied by AdaBoost.M1. Experiment results are tabulated in Table 7.

Table 7. Performance of C4.5 \& AdaBoost.M1

\begin{tabular}{cccc}
\hline \hline Measure & Class & C4.5 & AdaBoost.M1 \\
\hline F & C1 & 0.9398 & 0.9344 \\
Measure & C2 & 0.8625 & 0.8831 \\
& C3 & 0.8504 & 0.8197 \\
\hline Accuracy & & 0.9157 & 0.9112 \\
G-mean & & 0.8538 & 0.8661 \\
\hline \hline
\end{tabular}

Respecting to classification performance of $\mathrm{C} 4.5$, performance on class $\mathrm{C} 1$ is significantly better than the other two small classes C2 and C3. By applying AdaBoost.M1, performance of class $\mathrm{C} 1$ remains the similar value; that of class $\mathrm{C} 2$ is improved by $2.06 \%$; and that of class $\mathrm{C} 3$ is decreased by $3.13 \%$; Classification accuracy of C4.5 does not change a lot and G-mean value is slightly improved by $1.23 \%$. Based on these observations, we set up two learning objectives: 1) to further balance the identify ability on each class; and 2) to improve the recognition ability of class $\mathrm{C} 3$.

Respecting to the first learning objective, the fitness function is G-mean evaluation. The resulting prototype of a cost vector is $\left[\begin{array}{lll}0.4206 & 0.6256 & 1.0000\end{array}\right]$. Integrating this cost vector into AdaC2.M1, classification performance of each individual class reported by recall value and the overall performance evaluated by G-mean, together with those yielded by $\mathrm{C} 4.5$ and $\mathrm{C} 4.5$ applied by AdaBoost.M1 are tabulated in Table 8 . Recall value of class $\mathrm{C} 1$ is decreased by both AdaBoost.M1 and AdaC2.M1, but still keeps a good performance. Recall value of C2 is increased by $5.84 \%$ through applying Adaboost.M1 and by $9.49 \%$ through applying AdaC2.M1. For the class C3, recall value of $\mathrm{C} 4.5$ fails to be improved by AdaBoost.M1 and is increased 
by $9.08 \%$ through applying AdaC2.M1. G-mean value of C4.5 is slightly improved by applying AdaBoost.M1 and significantly improved by $5.75 \%$ by applying AdaC2.M1. AdaC2.M1 achieves the best G-mean value by increasing recall values of both classes $\mathrm{C} 2$ and $\mathrm{C} 3$.

Table 8. G-mean Evaluation

\begin{tabular}{cccc}
\hline \hline Class & C4.5 & AdaBoost.M1 & AdaC2.M1 \\
\hline C1(R) & 0.9703 & 0.9447 & 0.9106 \\
C2(R) & 0.8229 & 0.8813 & 0.9178 \\
C3(R) & 0.7966 & 0.7955 & 0.8874 \\
G-mean & 0.8539 & 0.8661 & 0.9014 \\
\hline \hline
\end{tabular}

Respecting to the second learning objective, the fitness function is the F-measure evaluation of class C3. The resulting prototype cost vector is [0.7697 0.98041 .0000$]$. Integrating this cost vector into AdaC2.M1, classification performance of class C3 together with those of C4.5 and C4.5 applied by AdaBoost.M1 are stated in Table 9. The Fmeasure performance of $\mathrm{C} 4.5$ is decreased by applying AdaBoost.M1 since the recall value is not changed a lot and precision value is lowered. By applying AdaC2.M1, recall value is increased and precision value is lowered such that these two values get closer. The resulting F-measure value obtained by AdaC2.M1 is a little bit better than that of $\mathrm{C} 4.5$.

Table 9. F-measure Evaluation on Class C3

\begin{tabular}{cccc}
\hline \hline & C4.5 & AdaBoost.M1 & AdaC2.M1 \\
\hline $\mathrm{R}$ & 0.7966 & 0.7955 & 0.8763 \\
$\mathrm{P}$ & 0.9467 & 0.8574 & 0.8547 \\
$\mathrm{~F}$ & 0.8504 & 0.8197 & 0.8613 \\
\hline \hline
\end{tabular}

\subsection{Nursery Database}

Nursery Database was derived to rank applications for nursery schools. There are 12960 instances, each described by 8 nominal attributes. The original data has 5 classes. Since one class, "recommend", has only 2 instances, we therefore combine this class with class "very-recommend". Table 10 describes the class distribution.

Table 10. Class Distribution

\begin{tabular}{cccc}
\hline \hline index & class name & class size & class distribution \\
\hline C1 & not-recom & 4320 & $33.33 \%$ \\
C2 & very-recom & 330 & $2.55 \%$ \\
C3 & priority & 4266 & $32.92 \%$ \\
C4 & spec-prior & 4044 & $31.20 \%$ \\
\hline \hline
\end{tabular}

With this data set, class $\mathrm{C} 2$ is the only small class. Experiment results of C4.5 and C4.5 applied by AdaBoost.M1. are tabulated in Table 11.

\section{Table 11. Performance of C4.5 \& Ad- aBoost.M1}

\begin{tabular}{cccc}
\hline \hline Measure & Class & C4.5 & AdaBoost.M1 \\
\hline F & C1 & 1 & 1 \\
Measure & C2 & 0.7784 & 0.8992 \\
& C3 & 0.9617 & 0.9829 \\
& C4 & 0.9765 & 0.9895 \\
\hline Accuracy & & 0.9747 & 0.9887 \\
G-mean & & 0.9250 & 0.9625 \\
\hline \hline
\end{tabular}

Obviously, performance of class $\mathrm{C} 2$ is the worst among these 4 classes by both $\mathrm{C} 4.5$ and AdaBoost.M1, even thought it is improved by applying AdaBoost.M1. Classification accuracy and G-mean value of $\mathrm{C} 4.5$ are improved by applying AdaBoost.M1. We set up two learning objectives: 1) to further balance the identify ability on each class; and 2) to improve the recognition ability of class $C 2$.

Respecting to the first learning objective, the fitness function is G-mean evaluation. The resulting prototype of a cost vector is $\left[\begin{array}{llll}0.7072 & 10.4888 & 0.6516\end{array}\right]$. Integrating this cost vector into AdaC2.M1, classification recall value of each individual class and the G-mean value, together with those generated by $\mathrm{C} 4.5$ and $\mathrm{C} 4.5$ applied by AdaBoost.M1 are tabulated in Table 12. Respecting to class $\mathrm{C} 2$, recall value is increased by $10.40 \%$ through applying AdaBoost.M1 and by $15.68 \%$ through applying AdaC2.M1. The G-mean value achieved by $\mathrm{C} 4.5$ is increased by $3.75 \%$ through applying AdaBoost.M1 and by $4.95 \%$ through applying AdaC2.M1. Both improvements are achieved mainly through increasing the recall value of class $\mathrm{C} 2$.

Table 12. G-mean Evaluation

\begin{tabular}{cccc}
\hline \hline Class & C4.5 & AdaBoost.M1 & AdaC2.M1 \\
\hline C1(R) & 1 & 1 & 1 \\
C2(R) & 0.7776 & 0.8816 & 0.9344 \\
C3(R) & 0.9630 & 0.9851 & 0.9755 \\
C4(R) & 0.9750 & 0.9889 & 0.9906 \\
G-mean & 0.9250 & 0.9625 & 0.9745 \\
\hline \hline
\end{tabular}

Respecting to the second learning objective, the fitness function is the F-measure evaluation of class $\mathrm{C} 2$. The resulting prototype cost vector is [ $\left[\begin{array}{lllll}0.7131 & 1 & 0.5310 & 0.2895\end{array}\right]$. Integrating this cost vector into AdaC2.M1, classification performance of class $\mathrm{C} 2$ together with those of $\mathrm{C} 4.5$ and C4.5 applied by AdaBoost.M1 are stated in Table 13. By applying AdaBoost.M1, F-measure value of C4.5 is significantly improved, from $77.84 \%$ to $89.92 \%$. Both recall and 
precision values are increased simultaneously. By applying AdaC2.M1, recall value is further improved and precision value is slightly decreased comparing with that of AdaBoost.M1. The combination evaluation F-measure of AdaC2.M1 is better than that of AdaBoost.M1.

\section{Table 13. F-measure Evaluation on Class C3}

\begin{tabular}{cccc}
\hline \hline & C4.5 & AdaBoost.M1 & AdaC2.M1 \\
\hline $\mathrm{R}$ & 0.7809 & 0.8816 & 0.9371 \\
$\mathrm{P}$ & 0.7776 & 0.9191 & 0.9043 \\
$\mathrm{~F}$ & 0.7784 & 0.8992 & 0.9197 \\
\hline \hline
\end{tabular}

\section{Conclusion}

In this paper, we developed a cost-sensitive boosting algorithm AdaC2.M1 to tackle the class imbalance problem with multiple classes (more than two classes). The main contributions of this research are: 1) to the best of our knowledge, this is the first work to address the class imbalance problem involving multiple classes. The significant hardness and importance in solving the class imbalance problem attracts a lot of research interests. However, most the existing approaches assume a bi-class setting. Due to the complicated situations when multiple classes present, methods for bi-class problems are not directly applicable; 2) the AdaC2.M1 algorithm has been developed by reducing its weight update parameter to minimize the overall training error of the combined classifier taking the misclassification costs into consideration. This process is crucial for the boosting efficiency; 3) our study shows that AdaC2.M1 is capable to adjust the data distributions and bias the leaning focuses among classes by setting up different cost values; and 4) we set up efficient cost vectors for applying AdaC2.M1 by the searching of the Genetic Algorithm. Conducted experimental tests on three "real world" data sets indicate that, with the searching results of GA, AdaC2.M1 is capable to improve the base classification's performances and accomplish better results than AdaBoost.M1 when both boosting algorithms are applied to the $\mathrm{C} 4.5$ classification systems. Due to the nature of GA, searching of cost setups might be time-consuming with some applications. This approach is still respectable considering that this searching is usually an off-line procedure such that the learning speed is not a crucial issue.

\section{References}

[1] A. P. Bradley. The use of the area under the ROC curve in the evaluation of machine learning algorithms. Pattern Recognition, 30(7):1145-1159, 1997.

[2] N. Chawla, N. Japkowicz, and A. Kolcz. Editorial: Special issue on learning from imbalanced data sets. SIGKDD Explorations Special Issue on Learning from Imbalanced Datasets, 6(1):1-6, 2004.
[3] C. Elkan. The foundations of cost-sensitive learning. In Proceedings of the Seventeenth International Joint Conference on Artificial Intelligence, pages 973-978, Seattle, Washington, August 2001.

[4] W. Fan, S. J. Stolfo, J. Zhang, and P. K. Chan. Adacost:misclasification cost-sensitive boosting. In Proceedings of Sixth International Conference on Machine Learning(ICML-99), pages 97-105, Bled, Slovenia, 1999.

[5] Y. Freund and R. E. Schapire. A decision-theoretic generalization of on-line learning and an aplication to boosting. Journal of Computer and System Sciences, 55(1):119-139, August 1997.

[6] J. Friedman, T. Hastie, and R. Tibshirani. Additive logistic regression: a statistical view of boosting. Annals of Statistics, 28(2):337374, April 2000.

[7] J. H. Holland. Adaptation in Natural and Artificial Systems. University of Michigan Press, Ann Arbor, MI, 1975.

[8] N. Japkowicz. Supervised versus unsupervised binary-learning by feedforward neural networks. Machine Learning, 41(1), 2001.

[9] N. Japkowicz and S. Stephen. The class imbalance problem: A systematic study. Intelligent Data Analysis Journal, 6(5):429-450, November 2002

[10] M. V. Joshi. Learning Classifier Models for Predicting Rare Phonemena. PhD thesis, University of Minnesota, Twin Cites, Minnesota, USA, 2002.

[11] M. V. Joshi, V. Kumar, and R. C. Agarwal. Evaluating boosting algorithms to classify rare classes: Comparison and improvements. In Proceeding of the First IEEE International Conference on Data Mining(ICDM'01), 2001.

[12] M. Kubat, R. Holte, and S. Matwin. Machine learning for the detection of oil spills in satellite radar images. Machine Learning, 30:195215, 1998.

[13] D. Lewis and W. Gale. Training text classifiers by uncertainty sampling. In Proceedings of the Seventeenth Annual International ACM SIGIR Conference on Research and Development in Information, pages 73-79, New York, NY, August 1998.

[14] P. M. Murph and D. W. Aha. UCI Repository Of Machine Learning Databases. Dept. Of Information and Computer Science, Univ. Of California: Irvine, 1991.

[15] M. Pazzani, C. Merz, P. Murphy, K. Ali, T. Hume, and C. Brunk. Reducing misclassification costs. In Proceedings of the Eleventh International Conference on Machine Learning, pages 217-225, New Brunswick, NJ, July 1994.

[16] F. Provost and T. Fawcett. Analysis and visualization of classifier performance: Comparison under imprecise class and cost distributions. In Proceedings of the Third International Conference on Knowledge Discovery and Data Mining (KDD-97), pages 43-48, Newportbeach, CA, August 1997.

[17] J. R. Quinlan. C4.5: programs for machine learning. Morgan Kaufmann Publishers, 1993.

[18] R. E. Schapire and Y. Singer. Boosting the margin: A new explanation for the effectiveness of voting methods. Machine Learning, 37(3):297-336, 1999.

[19] R. E. Schapire and Y. Singer. Improved boosting algorithms using confidence-rated predictions. Machine Learning, 37(3):297-336, 1999.

[20] Y. Sun, A. K. C. Wong, and Y. Wang. Parameter inference of costsensitive boosting algorithms. In Proceedings of 4th International Conference on Machine Learning and Data Mining in Pattern Recognition, pages 21-30, Leipzig, Germany, July 2005.

[21] K. M. Ting. A comparative study of cost-sensitive boosting algorithms. In Proceedings of the 17th International Conference on Machine Learning, pages 983-990, Stanford University, CA, 2000.

[22] K. M. Ting. An instance-weighting method to induce cost-sensitive trees. IEEE Transaction on Knowledge and Data Engineering, 14(3):659-665, 2002.

[23] G. Weiss. Mining with rarity: A unifying framework. SIGKDD Explorations Special Issue on Learning from Imbalanced Datasets, 6(1):7-19, 2004.

[24] G. Weiss and F. Provost. Learning when training data are costly: The effect of class distribution on tree induction. Journal of Artificial Intelligence Research, 19:315-354, 2003. 